SOME NOTES ON THE PLACEMENT OF CORRECTORS

BNL/SNS TECHNICAL NOTE

NO. 040

\author{
C. J. Gardner
}

December 29, 1997

ALTERNATING GRADIENT SYNCHROTRON DEPARTMENT

BROOKHAVEN NATIONAL LABORATORY

UPTON, NEW YORK 11973 


\title{
Some Notes on the Placement of Correctors
}

\author{
C.J. Gardner
}

December 29, 1997

Following are some notes pertaining to the placement of correction elements in the SNS Ring.

\section{SNS Lattice and Correction Elements}

The SNS ring lattice [1-4] consists of four superperiods, each containing a $90^{\circ}$ arc and a long straight section. The four superperiods are labeled $A$, $\mathrm{B}, \mathrm{C}, \mathrm{D}$ and run sequentially along the beam direction from the beginning of one arc to the next. The order of magnets in each superperiod $\mathrm{X}$ is DHX1, QVX1, DHX2, QHX2, ..., DHX8, QHX8, QVX9, QHX10, QVX11, QHX12, where D and Q denote dipoles and quadrupoles, and $\mathrm{H}$ and $\mathrm{V}$ refer to the horizontal and vertical planes. The long straight section in each superperiod runs from QHX8 through QHX12.

To compensate for the small unavoidable field imperfections and magnet alignment errors that can lead to resonance excitation, the ring will have a set of correction elements consisting of horizontal and vertical dipoles, skew quadrupoles, sextupoles, skew sextupoles and octupoles. Trim windings on the lattice quadrupoles will allow for any necessary quadrupole corrections. Correction dipoles and skew quadrupoles will be mounted downstream of the position monitor at each quadrupole with the dipole and skew quadrupole windings wound on the same core. We shall use QSX1, QSX2, ..., QSX11, QSX12 to label the skew quadrupoles downstream of quadrupoles QVX1, QHX2,..., QVX11, QHX12 respectively. Sextupoles called SHX8 and SVX9 will be placed downstream of quadrupoles QHX8 and QVX9 in each superperiod; similarly, skew sextupoles (SSVX11 and SSHX12) will be placed downstream of QVX11 and QHX12. 


\section{Resonance Excitation}

Transverse resonances [5] are defined by the equation

$$
m Q_{x}+n Q_{y}=N
$$

where $Q_{x}$ and $Q_{y}$ are the horizontal and vertical tunes, and $m, n$, and $N$ are integers. If $m$ and $n$ have opposite signs, the resonance is called a difference resonance; otherwise it is called a sum resonance. The order, $l$, of the resonance is

$$
l=|m|+|n| .
$$

The resonance condition (1) arises from the first-order perturbation treatment of the vector potential terms $x^{|m|} y^{|n|}$ associated with the $2 l$-pole magnetic field. Resonance excitation occurs when the tunes are sufficiently close to the resonance and the excitation coefficient,

$$
\kappa=\int_{0}^{2 \pi r} \beta_{x}^{|m / 2|} \beta_{y}^{|n / 2|} K(s) e^{i \psi} d s,
$$

is nonzero. Here

$$
\begin{gathered}
\psi(s)=m\left(\mu_{x}-Q_{x} \theta\right)+n\left(\mu_{y}-Q_{y} \theta\right)+N \theta, \quad \theta=s / r \\
\mu_{x}(s)=\int_{0}^{s} \frac{d s^{\prime}}{\beta_{x}\left(s^{\prime}\right)}, \quad \mu_{y}(s)=\int_{0}^{s} \frac{d s^{\prime}}{\beta_{y}\left(s^{\prime}\right)}, \quad K(s)=\frac{e}{c p}\left(\frac{\partial^{(l-1)} B}{\partial x^{(l-1)}}\right),
\end{gathered}
$$

and $B=B_{y}\left(B_{x}\right)$ if $n$ is even (odd). Since

$$
\psi(s) \approx m \mu_{x}+n \mu_{y} \approx N \theta,
$$

when the tunes are near the resonance, we see that the excitation coefficient is essentially the $N$ th harmonic, in azimuth $\theta$, of the $2 l$-pole strength $K(s)$. Thus if the tunes are sufficiently close to the resonance and if $n$ is even (odd), then the resonance will be excited by the $N$ th harmonic of the normal (skew) $2 l$-pole fields present in the machine.

\section{Resonance Correction}

For the correction of the $m Q_{x}+n Q_{y}=N$ resonance we require

$$
\kappa_{0}+\kappa=0
$$


where $\kappa_{0}$ is the excitation coefficient due to imperfections in the machine, and $\kappa$ is that produced by the resonance correction scheme. Note that the excitation coefficient is in general complex, so correction of a given resonance requires cancellation of both the real and imaginary parts of $\kappa_{0}$. For a set of identical thin correction elements we have

$$
\kappa=K \sum_{j} I_{j} \beta_{x j}^{|m / 2|} \beta_{y j}^{|n / 2|} e^{i \psi_{j}}
$$

where $K$ is the integrated strength per unit current of each correction element, $I_{j}$ is the current in the $j$ th element, and

$$
\beta_{x j}=\beta_{x}\left(s_{j}\right), \quad \beta_{y j}=\beta_{y}\left(s_{j}\right), \quad \psi_{j}=\psi\left(s_{j}\right)
$$

\section{Corrector Phases}

Effective correction of a given resonance requires careful consideration of the betatron phase advances between correctors. Suppose we have two identical correctors located at $s_{1}$ and $s_{2}$ in a ring consisting of $M$ superperiods. Then for each corrector we have

$$
\psi_{i}=m\left(\mu_{x i}-Q_{x} \theta_{i}\right)+n\left(\mu_{y i}-Q_{y} \theta_{i}\right)+N \theta_{i}
$$

or

$$
\psi_{i}=m \mu_{x i}+n \mu_{y i}+\left(N-m Q_{x}-n Q_{y}\right) \theta_{i}
$$

where

$$
\psi_{i}=\psi\left(s_{i}\right), \quad \mu_{x i}=\mu_{x}\left(s_{i}\right), \quad \mu_{y i}=\mu_{y}\left(s_{i}\right), \quad \theta_{i}=s_{i} / r
$$

Thus

$$
\psi_{2}-\psi_{1}=m\left(\mu_{x 2}-\mu_{x 1}\right)+n\left(\mu_{y 2}-\mu_{y 1}\right)+\left(N-m Q_{x}-n Q_{y}\right)\left(\theta_{2}-\theta_{1}\right) .
$$

Now, if $s_{1}$ and $s_{2}$ are separated by $k$ superperiods, then

$$
\begin{aligned}
& \mu_{x 2}-\mu_{x 1}=2 \pi Q_{x} k / M=Q_{x}\left(\theta_{2}-\theta_{1}\right) \\
& \mu_{y 2}-\mu_{y 1}=2 \pi Q_{y} k / M=Q_{y}\left(\theta_{2}-\theta_{1}\right)
\end{aligned}
$$

and (13) becomes

$$
\psi_{2}-\psi_{1}=N\left(\theta_{2}-\theta_{1}\right)=2 \pi k N / M
$$


Here we see that $\psi_{2}-\psi_{1}$ is independent of $\mu_{x}, \mu_{y}$ and the machine tunes; it depends only on the azimuthal separation $\theta_{2}-\theta_{1}$. Thus, by taking advantage of the superperiod symmetry we can produce azimuthal harmonics that are independent of the machine tunes. Moreover, we can use the symmetry to restrict which harmonics are produced. The simplest example of this is the placement of two correctors such that $\theta_{2}-\theta_{1}=\pi$ in a ring with an even number of superperiods; if the correctors are excited with equal currents of the same (opposite) polarity then only even (odd) harmonics are produced. If we add more correctors, we can further restrict the harmonics. For example, if the number of superperiods is divisible by 4 (as it is for the SNS ring), then 4 equally spaced correctors separated by $\pi / 2$ in azimuth and excited with equal currents of the same polarity will produce only harmonics $0,4,8,12$, and so on. If this same set is excited with equal currents of alternating polarities, then it will produce only harmonics 2, 6, 10,14, and so on. In general, the more superperiods one has in a lattice, the more one can restrict the harmonics by adding correctors; in this way one can create a relatively pure harmonic for the desired resonance correction.

From (16) we also see that if $2 \pi k N / M$ is an odd multiple of $\pi / 2$, then the two correctors will provide orthogonal control of the real and imaginary parts of the resonance excitation coefficient; this will again be independent of the machine tunes. In the SNS ring we have $M=4$ and correctors placed one superperiod apart will therefore provide orthogonal control of any resonance for which $N$ is odd.

The lattice may have other symmetries or approximate symmetries that one can exploit. In a FODO lattice with a betatron phase advance of approximately $\pi / 2$ per cell, the phase advance between two correctors placed $l$ cells apart is approximately $l \pi / 2$ :

$$
\mu_{x 2}-\mu_{x 1}=l \pi / 2, \quad \mu_{y 2}-\mu_{y 1}=l \pi / 2 .
$$

Then (13) becomes

$$
\psi_{2}-\psi_{1}=m(l \pi / 2)+n(l \pi / 2)+\left(N-m Q_{x}-n Q_{y}\right)\left(\theta_{2}-\theta_{1}\right),
$$

and for $N-m Q_{x}-n Q_{y}=0$ we have

$$
\psi_{2}-\psi_{1}=m(l \pi / 2)+n(l \pi / 2) .
$$

Here we get a phase difference that is some multiple of $\pi / 2$ provided the tunes satisfy (or nearly satisfy) the resonance condition. If the phase 
difference is an even (odd) multiple of $\pi$, then the two correctors will affect the resonance only if $I_{2} \neq-I_{1}\left(I_{2} \neq I_{1}\right)$. If the phase difference is an odd multiple of $\pi / 2$, then the two correctors will again provide orthogonal control of the real and imaginary parts of the resonance excitation coefficient.

\section{$5 \quad$ Beta Function Considerations}

Effective correction of a given resonance also requires that the correctors be placed as close as possible to locations where the beta function is large. This follows directly from (8) which shows that the correction due to the $j$ th element in a correction scheme is proportional to $\beta_{x j}^{|m / 2|} \beta_{y j}^{|n / 2|}$. Simultaneous correction of two resonances excited by the same magnetic multipole generally requires one set of correction elements placed where $\beta_{x}$ is large and another set placed where $\beta_{y}$ is large. This is the case for the $2 Q_{x}=N$ and $2 Q_{y}=N$ resonances, the $3 Q_{x}=N$ and $Q_{x}+2 Q_{y}=N$ resonances, and the $3 Q_{y}=N$ and $Q_{y}+2 Q_{x}=N$ resonances.

\section{References}

1. W.T. Weng, et. al., "Accumulator Ring Design for the NSNS Project", Proceedings of the 1997 Particle Accelerator Conf., Vancouver, B.C., Canada, May 12-16, 1997.

2. Y.Y. Lee, "The 4 Fold Symmetric Lattice for the NSNS Accumulator Ring”, BNL/NSNS Tech. Note No. 026, February 19, 1997.

3. C.J. Gardner, Y.Y. Lee, and A.U. Luccio, "Accumulator Ring Lattice for the National Spallation Neutron Source", Proceedings of the 1997 Particle Accelerator Conf., Vancouver, B.C., Canada, May 12-16, 1997.

4. C.J. Gardner, "Some Notes on Tuning the NSNS Ring Lattice", BNL/NSNS Tech. Note No. 037, August 12, 1997.

5. G. Guignard, "The General Theory of all Sum and Difference Resonances in a Three-Dimensional Magnetic Field in a Synchrotron", CERN 76-06, 23 March 1976; "A General Treatment of Resonances in Accelerators" CERN 78-11, 10 November 1978. 